\title{
An Improved Algorithm of Spatial Difference MUSIC for Direction of Arrival Estimation in a Smart Antenna
}

\author{
Li Bo ${ }^{1,2}$, Tingting $\mathrm{Li}^{1}, \mathrm{Li} \mathrm{Li}^{2}$, Yang $\mathrm{Hui}^{2}$ and Chuanlai Yuan ${ }^{3, *}$ \\ ${ }^{I}$ School of Information Engineering, East China Jiaotong University, Nanchang, 330013, China; ${ }^{2}$ Key Laboratory Ad- \\ vanced Control \& Optimization of Jiangxi Province, Nanchang, 330013, China; ${ }^{3}$ College of Electrical and Information \\ Engineering, Hunan University of Technology, Zhuzhou, 412007, PR China
}

\begin{abstract}
The estimation of direction of arrival (DOA) of a smart antenna is not able to perform high resolution in a mixed noise field with high correlation. In this paper, a modified multiple signal classification (MUSIC) algorithm based on spatial difference is proposed for improving the resolution of DoA estimation. The algorithm can eliminate the effect of mixed noise through computing the difference of the output signal covariance matrix and resolving the uncorrelated and coherent signal respectively. In order to obtain the orthogonal subspaces of the signal and noise, the Toeplitz decomposition and singular value decomposition (SVD) algorithm are applied to reconstruct the matrix. Accordingly, the MUSIC algorithm is adopted to estimate the DOA efficiently. In our research, several contrastive simulations corresponding to DOA estimation between the traditional MUSIC algorithm and the proposed method are implemented and analyzed. The results demonstrate the feasibility and high efficiency of our proposed algorithm.
\end{abstract}

Keywords: DOA estimation, MUSIC, smart antenna, spatial difference.

\section{INTRODUCTION}

With widely application of personal communications services, how to logically allocate the limited spectrum resource has become an important issue. By introducing the fourth dimension multiple access, smart antenna can differentiate signals with space propagation direction under the circumstances with the same time, the same frequency and the same address code, and consequently improve the utilize of spectrum resource [1]. As an adaptive array signal processing technique, the direction of arrival (DOA) in a smart antenna becomes one of the vital problems to be resolved [2$3]$.

Generally speaking, a majority of researches on DOA estimation are carried out under the assumption of white noise [4-8] For example, Capon, linear prediction approach, MUSIC, ESPRIT. Unfortunately, the signals of target sources in smart antenna are suffered from the effects of multiple paths, diffraction in wave propagation, color noise and mixed noise interference. Accordingly, the DOA estimation in mixed noises fields of coherent array sources becomes intensive topics in recent years. [9-10] Considering the problem of narrowband DOA estimation under spatially colored noises, a new method for maximum likehood DOA estimation of stochastic and mixed signals was proposed [11-12] presented a spatial smoothing with improved aperture method to improve the effective array aperture ; [13] presented an algorithm that divided the array covariance matrix into Toeplitz structure and non-Toeplitz structure matrix, then cancelled color noises by utilizing matrix difference translation method; [14-17] presented multiple signal classification (MUSIC) method based on eigen-decomposition of received signal correlation matrix, on the assuming of enough precise of model, the DOA estimation could achieve arbitrary high resolution theoretically. As mentioned above, currently a majority of DOA estimating algorithms only take into account of Gaussian white noises or some customizing noise fields, and do not give consideration of both coherent and uncorrelated sources for signals recognition in mixed noises fields.

To analyze the mixed noise of DOA estimation, a novel DOA estimation algorithm which combined with Toeplitz and Svd methods is proposed and satisfies the high resolution DOA estimating under high correlated, low SNR and near distance sources in mixed noises fields. Based on the MUSIC algorithm, a matrix difference method which can individually recognize uncorrelated and correlated (coherent) sources is also presented. Besides, a phenomenon of fact that conventional MUSIC method would mistakenly estimate DOA in mixed noises fields is detected by a number of simulation and numeric analysis.

\section{DOA ESTIMATION MODEL OF SMART ANTEN- NA}

The configuration of smart antenna $[1,2]$ is shown in the following Fig. (1).

As shown in Fig. (1) that the input signals can be treated as planar waves, so the phase differences are uniquely determined by carrier wavelength, angle of arrive and the space distribution of antenna. As for the elements in a smart antenna, carrier wavelength and the space distribution are almost 
the same, whereas the signal intensity of array is distinctly different since the different angles of arrival of input signal induce phase difference. Hence, the problem of DOA estimation plays an important role in smart antenna processing.

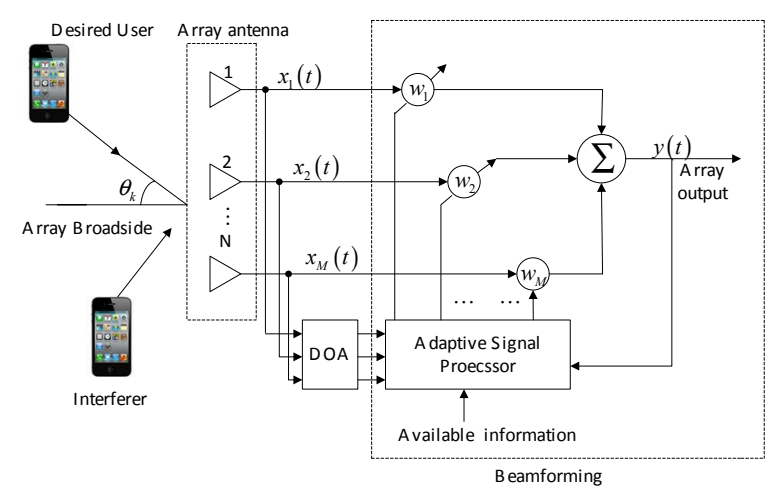

Fig. (1). A functional block diagram of a smart antenna system.

The DOA estimation Model of target sources in mixed noises fields is given as the following Fig. (2).

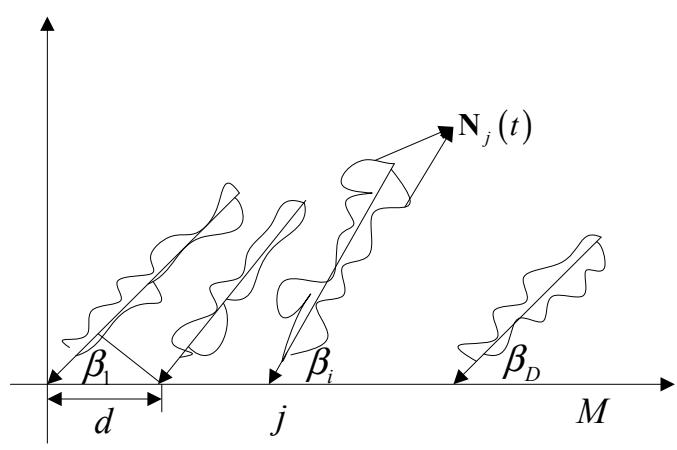

Fig. (2). The DOA estimation model of distributed target sources.

In Fig. (2), $\beta_{i}$ is steering vector of the $i$ th target signal source, and $D$ is the quantity of target signal sources. The uniform linear array is consisted of equally spaced $M(M>D)$ elements, which have the equal behavior to isotropy in direction and equal distance $d$, whose value is less than half of signal wavelength with the max frequency.

When the target signals arrival at the array, the jth element output vector can be represented as the following form:

$\mathbf{x}_{j}(t)=\sum_{i=1}^{D} \int_{-\pi}^{\pi} \boldsymbol{\alpha}(\beta) s_{i}\left(\beta-\beta_{i}, t\right) d \beta+\mathbf{N}_{j}(t)$

Where $s_{i}\left(\beta-\beta_{i}, \mathrm{t}\right)$ is the direction signal density function of $i$ th target signal source at time $t$ and $\mathbf{N}_{j}(t)$ is the mixed noise vector. In the application environment of smart antenna the radiating signals of different distributed source commonly are coherent.

Based on the theory of Schwartz Inequation, the difference between the two signals is a constant complex value when they are interfered, and the parameter $s_{i}(\beta-\beta, \mathrm{t})$ in formula (1) can be revised as following formula (2): $s_{i}\left(\beta-\beta_{i}, \mathrm{t}\right)=s_{i}(t) g_{i}\left(\beta-\beta_{i}\right)$

where $g_{i}\left(\beta-\beta_{i}\right)$ is the signal angle distribution function of the $i$ th target source, and it is a deterministic function centered with $\beta_{i}$, and parameter $g_{i}\left(\beta-\beta_{i}\right)$ satisfies with:

$\int_{-\pi}^{\pi} g_{i}\left(\beta-\beta_{i}\right) d \beta=1$

And the received signals model can be expressed as:

$\mathbf{X}(t)=\mathbf{B S}(t)+\mathbf{N}(t)$

\section{THE SPATIAL DIFFERENCE MUSIC ALGO- RITHM FOR DOA ESTIMATION}

\subsection{Spatial Difference Algorithm}

Spatial difference algorithm is applied to eliminate the effects of mixed noises in the covariance matrix [18]. Based on formula (4) the covariance matrix of receiving data can be written as:

$$
\mathbf{R}=E\left\{\mathbf{X}(t) \mathbf{X}(t)^{H}\right\}=\mathbf{R}_{T}+\mathbf{R}_{N T}+\mathbf{Q}_{T}
$$

Where $\mathbf{R}_{T}$ is the correlation matrix of uncorrelated sources, which is a Hermite-Toeplitz matrix; $\mathbf{R}_{N T}$ is the correlation matrix of the correlated or coherent sources, which is a Hermite matrix but not a Toeplitz matrix; $\mathbf{Q}_{T}$ is the covariance matrix of mixed noises, and it is Hermite-Toeplitz matrix on the supposing in the presence of stationary and correlation noise fields.

Definition 1: Suppose the matrix $\mathbf{E}$ is in Toeplitz form and $\mathbf{J}$ represents the exchange matrix, the following formulation is satisfied:

$$
\mathbf{J E}^{T} \mathbf{J}=\mathbf{E}
$$

Definition 2: The spatial difference matrix is formulated as:

$$
\mathbf{R}^{d}=\mathbf{R}-\mathbf{J} \mathbf{R}^{T} \mathbf{J}
$$

Theorem 1 :

If there exists several correlated, coherent and uncorrelated sources in the group of uniform linear distributed target sources environment, space difference matrix of signals does not contain information of the uncorrelated sources and it can cancel mixed noises in Toeplitz forms.

\section{Proof of theorem 1:}

Combining formula (5) and the definition (1), (2), we can get the following conclusion:

$$
\begin{aligned}
\mathbf{R}^{d} & =\mathbf{R}-\mathbf{J} \mathbf{R}^{T} \mathbf{J} \\
& =\mathbf{R}_{T}+\mathbf{R}_{N T}+\mathbf{Q}^{T}-\mathbf{J}\left(\mathbf{R}_{T}+\mathbf{R}_{N T}+\mathbf{Q}_{T}\right)^{T} \mathbf{J} \\
& =\mathbf{R}_{N T}-\mathbf{J}\left(\mathbf{R}_{N T}\right)^{T} \mathbf{J}
\end{aligned}
$$

From the formula (8) we can see that the difference matrix do not contain Toeplitz terms, which contains neither uncorrelated sources information, nor the mixed noises information. So the sources can be classified by types and 
identified respectively. Furthermore, it decreases the effect from mixed noises sources in DOA estimation.

Consequently, the estimation matrix $\wedge \mathbf{R}^{d}$ would come close to the actual covariance matrix $\mathbf{R}^{d}$ by Teoplitzing operation, namely :

$\min _{\mathbf{R} \in \mathbf{S}}\left\|\hat{\mathbf{R}}^{d}-\mathbf{R}^{d}\right\|$

Where $\mathbf{S}$ is the Toeplitz matrix set.

\subsection{Toeplitz Approximation Algorithm}

The essence of Toeplitz approximation algorithm is computing the average of oblique diagonal elements in covariance matrix, which can be described as the following formulas:

$$
\begin{aligned}
& r(-p)=\frac{1}{M-p} \sum_{i=1}^{m-p} r_{i(i+n)}, 0 \leq p<M \\
& r(p)=r_{(-p)}^{*}
\end{aligned}
$$

Where $r_{i j}$ is the element of the covariance matrix, and $M$ is the quantity of array elements. In the last the elements the matrix $\mathbf{R}^{d}$ is revised with $r_{i j}=r_{(i-j)}$, so the reconstructed covariance matrix $\mathbf{R X}$ have Toeplitz character that be utilized to realize uncorrelating and uninterference.

\subsection{Svd Algorithm}

The goal of Svd method is to decompose covariance matrix RX, and then achieve two orthogonal signal subspaces and mixed noises subspace which provide possibility for efficient utilization of MUSIC algorithm.

Step 1: On the assuming of above array model, the first procedure is decomposing the reconstructed covariance matrix $\mathbf{R X}$ as:

$$
[\mathbf{U}, \mathbf{S}, \mathbf{V}]=\operatorname{svd}(\mathbf{R X})
$$

Where $\mathbf{U}$ and $\mathbf{V}$ are two orthogonal matrices, which respectively represent signal subspace and noise subspace, and the matrix $\mathbf{S}$ is a diagonal matrix. So the eigenvector of the $\max$ noise eigenvalue can be get by $\mathbf{V}_{n}=\mathbf{U}(:, D+1: M)$.

Step 2: As to two correlated signal sources, the noise subspace vectors also contain some other eigenvectors besides vector $\mathbf{V}_{n}$. On the prediction of ensuring the Svd decomposing precision, adopt matrix low-rank approximation method to reconstruct a low-rank matrix RXX rather than decompose matrix $\mathbf{R X}$ with Svd method. Matrix $\mathbf{R X X}$ reconstructing as the following:

$$
\begin{gathered}
\mathbf{R X X}=\mathbf{U} * \mathbf{S S} * \mathbf{V} \\
\mathbf{S e t} \mathbf{S S}=\mathbf{S} \text { and } \\
\mathbf{S S}(M-1, M-1)=0 \\
\mathbf{S S}(M-2, M-2)=0 \\
\mathbf{S S}(M, M)=0
\end{gathered}
$$

The matrix RXX can be decomposed as following :

$$
[\mathbf{U U}, \mathbf{S S S}, \mathbf{V V}]=\operatorname{Svd}(\mathbf{R X X}, \mathbf{X})
$$

In the noises subspace, assuming $\mathbf{V}_{u u}$ to be the eigenvector set of eigenvalues except the max eigenvalue of $\mathbf{V}$, that is $\mathbf{V}_{u u}=\mathbf{U} \mathbf{U}(:, D+1: M)$.

Step 3: A mean-value scheme is adopted for the noises subspaces $\mathbf{V}_{n}$ and $\mathbf{V}_{u u}$ to achieve a high resolution of spatial spectrum estimation with the formula (15).

$$
\mathbf{V U}=\left(\mathbf{V}_{u u}+\mathbf{V}_{u}\right) / 2
$$

In the left of formula (15) matrix $\mathbf{V U}$ is the expected noise subspace.

\subsection{DOA Estimation Based on MUSIC Algorithm}

After processing of the previous three procedures, two orthogonal spaces of signal subspace and mixed noises subspace can be achieved by the distributed signal sources in mixed noises fields, then adopt MUSIC algorithm to realize high resolution DOA recognizing. In MUSIC, subspace decomposing method utilizes orthogonality between signal subspace and mixed noises subspace, to reconstruct spatial spectrum function that gives an indication of the angles of arrival based upon maxima vs. angle.

The procedures of DOA estimation are listed as the following:

Step 1: collecting the input sample $\mathbf{X}(i), i=1, \mathrm{~L}, N$, and estimating the covariance matrix of input signal as the following:

$$
\hat{\mathbf{R}_{\mathbf{X}}}=\frac{1}{N} \sum_{i=1}^{N} \mathbf{X}(i) \mathbf{X}^{H}(i)
$$

Step 2: Making use of the Toeplitz approximating algorithm, get the reconstructed covariance matrix RX which have the Toeplitz character.

Step 3: Taking utilization of Svd algorithm to decompose $\mathbf{R X}$ in two times, constructing high resolution spatial spectrum estimation of noise subspace $\mathbf{V U}$.

Step 4: On the basis of achieved noises subspace VU, adopt MUSIC algorithm to reconstruct spatial spectrum function, then estimate DOA values. Combining with formula (1), formula (2) and formula (4), we can see that the steering vectors related to signal vectors are orthogonal with eigenvectors of noises subspace, namely on the prediction that when the DOA estimating values of multiple path assumed to be $\beta_{i}$ it exist:

$$
\boldsymbol{\alpha}^{H}\left(\beta_{i}\right) \mathbf{V U}(\mathbf{V U})^{H} \boldsymbol{\alpha}\left(\beta_{i}\right)=0
$$

In the practical realization for the covariance matrices are achieved by the estimating data with finite observation, the estimating eigenvector of noise subspace will bring some errors during the covariance matrices being decomposed. So when errors is exist in VU the right of the formula (17) is not zero vector. Thereby, the DOA of multiple input signals can be recognized by estimating spectral peaks of MUSIC spatial spectrum, and the spectral peaks values can be computed as: 


$$
\begin{aligned}
\hat{\mathbf{P}}_{\text {MUSIC }}\left(\beta_{i}\right) & =\frac{1}{\left[\boldsymbol{\alpha}^{H}(\beta) \mathbf{V U}(\mathbf{V U})^{H} \boldsymbol{\alpha}(\beta)\right]} \\
& =\frac{1}{\left\|\boldsymbol{\alpha}^{H}(\beta) \mathbf{V U}\right\|^{2}}
\end{aligned}
$$

In the above formula (18) the DOA value is evaluated with the customizing spectrum peak $\beta_{i}$ value in the pattern.

\section{SIMULATIONS AND ANALYSIS}

\subsection{DOA Estimation Simulations and Analysis in Addi- tive Gaussian White Noise Fields}

In this section, the non-ideal environment exist additive Gaussian white noise with variance of 1.0 . We have performed three experiments to test the performance of our algorithm by contrast with the traditional MUSIC algorithm. Firstly, it is supposed that the presence of three moving target sources at azimuth angles $\left[-60^{\circ}, 40^{\circ}, 45^{\circ}\right]$ impinging on the array. In the experiment, the sources are non-correlated and the SNR is $10 \mathrm{~dB}$. The result pattern of this experiment is shown in the following Fig. (3a). In the second experiment, three moving target sources at azimuth angles $\left[-60^{\circ}, 30^{\circ}\right.$, $45^{\circ}$ ] impinging on the array, in which the moving targets with azimuth angles $\left[-60^{\circ}, 30^{\circ}\right]$ are highly correlated, and the SNR is also $10 \mathrm{~dB}$. Fig. (3b) is the result pattern of this experiment. Thirdly, we suppose that low SNR noncorrelated moving targets with SNR $5 \mathrm{~dB}$ at azimuth angles [ $\left.-13^{\circ},-10^{\circ}, 45^{\circ}\right]$ impinging on the array, and the result is shown in Fig. (3c).

From Fig. (3) we can observe that in the presence of additive Gaussian white noise fields MUSIC as well as our algorithm success to recognize DOA with non-correlated sources as shown in Fig. (3a), whereas MUSIC fail to recognize DOA for highly correlated sources and contrarily our algorithm success to estimate in the same case as shown in Fig. (3b). Lastly in the Fig. (3c), in the presence of near distance and with low SNR, in which the differences of the sources are inconspicuous and the SNR are $5 \mathrm{~dB}$, traditional MUSIC algorithm fail to estimate the multi-DOA with near angle distance, but our algorithm can effectively estimate the case.

\subsection{DOA Estimation Simulations and Analysis in Mixed Color Noises Fields}

Another scenario has also been tested for the proposed algorithm applied in the mixed color noises fields, where two interference sources of color noises are presented at background. The variances of the hypothesis noises are 2.25 and 3.24 , and the mean values of them are 1.0 and 1.2 respectively. Three experiments have also been performed to test the performance of our algorithm by contrast with the traditional MUSIC algorithm. In the first experiment, we suppose the presence of three moving targets which SNR is $10 \mathrm{~dB}$ and non-correlated at azimuth angles $\left[-60^{\circ}, 40^{\circ}, 45^{\circ}\right]$ impinging on the array. The result of this experiment is shown in Fig. (4a). Secondly highly correlated moving targets at azimuth angles $\left[-60^{\circ}, 30^{\circ}, 45^{\circ}\right]$ impinging on the array, and the SNR of them are $10 \mathrm{~dB}$. The result spectral pattern is shown in Fig. (4b). In the last experiment we suppose the azimuth differ- ences of the sources are inconspicuous and the SNR are $5 \mathrm{~dB}$, and the result spectral pattern is shown in the following Fig. $(4 \mathrm{c})$.

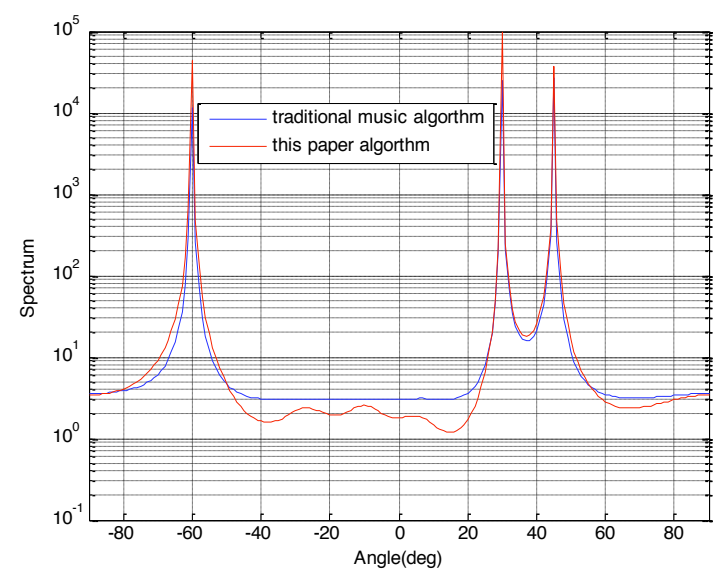

(a) DOA estimation spectrum pattern of non-correlation sources

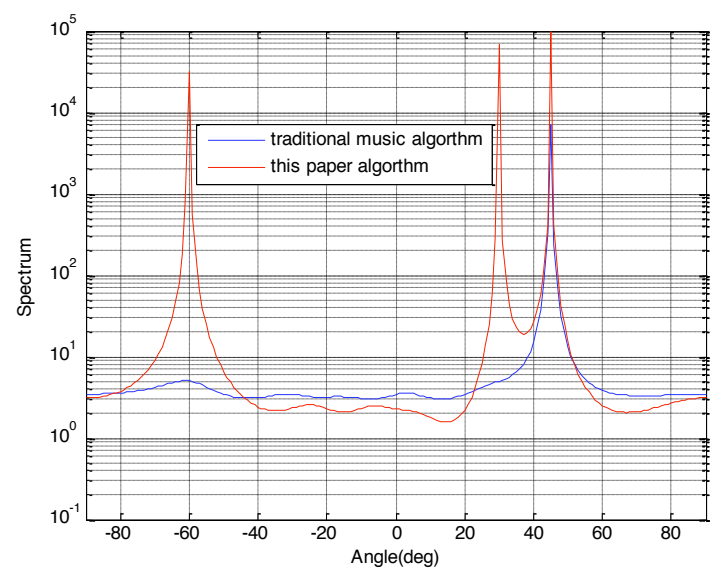

(b)DOA estimation spectrum pattern of high correlation sources

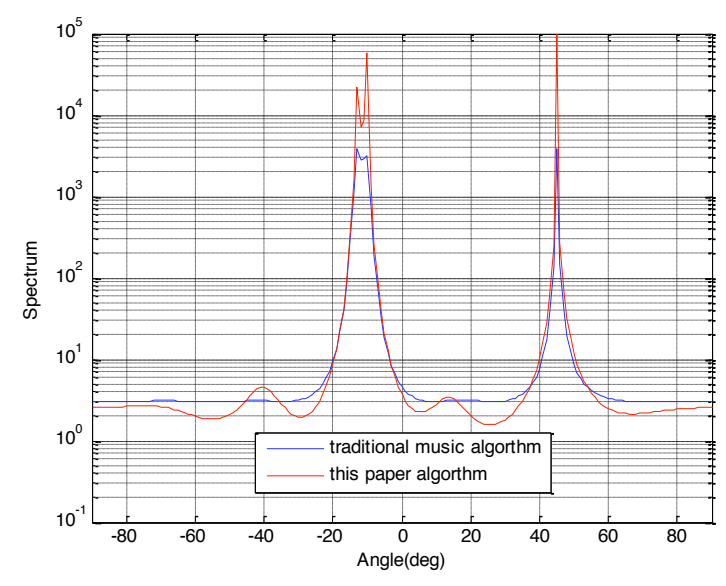

(c) DOA estimation spectrum pattern of near distance noncorrelation sources with low SNR noises

Fig. (3). Simulation results in additive Gaussian white noises fields. 


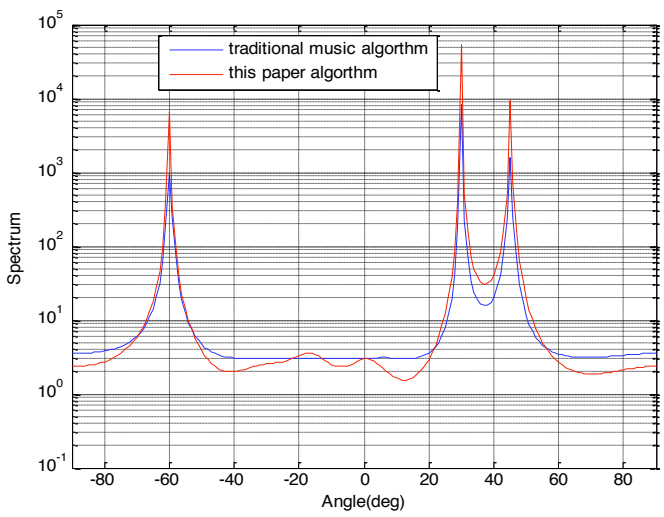

(a) DOA estimation spectrum pattern of non-correlation sources

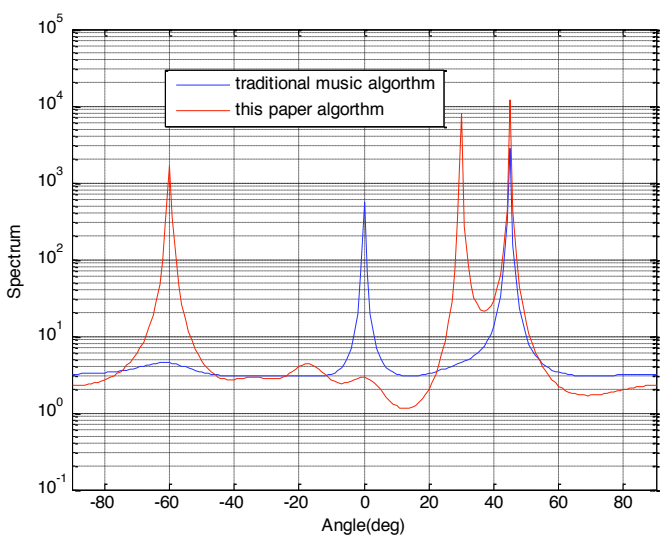

(b)DOA estimation spectrum pattern of high correlation sources

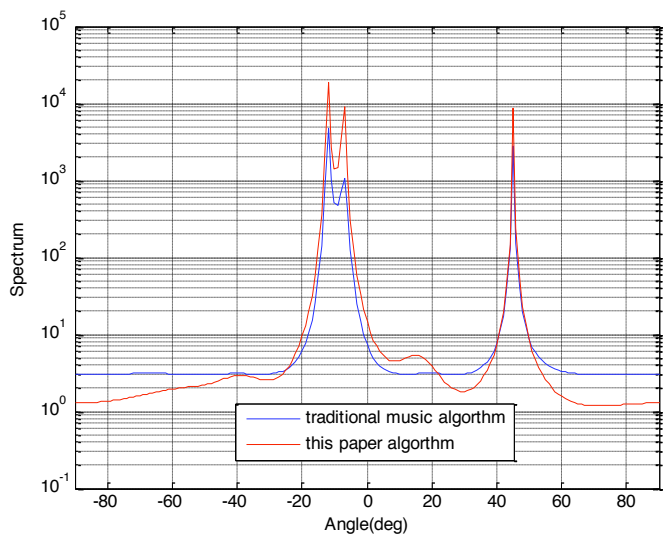

(c) DOA estimation spectrum pattern of near distance noncorrelation sources with low SNR noises

Fig. (4). Simulation results in mixed color noises fields.

In Fig. 4(a)-(c), we show the result patterns of angle and spectral power relation as function of the number of snapshots for the traditional algorithm and our algorithm for mixed color noises fields. From 4(a) we can see that in the background of mixed color noises the traditional MUSIC algorithm and our algorithm both can estimate the DOA values, and comparatively our algorithm can generate more sharp spectral peaks and lower sidelobes in the same SNR.
As for highly correlated sources simulation shown in Fig. 4(b). The last simulation for the scenario of unrelated near distance sources in space with low SNR environment, both the traditional MUSIC algorithm and the proposed algorithm can estimate the DOA of $45^{\circ}$, which is far away from the other sources and our algorithm have more sharp spectral peaks and lower sidelobes.

\section{CONCLUSION}

This paper proposed a novel spatial difference MUSIC (SDMUSIC) algorithm which adopts the method of difference reconstruction based on MUSIC method to resolve high-resolution DOA estimate in mixed noises fields. Simulation results confirm that our method can not only effectively resolve high resolution DOA estimation for uncorrelated sources with additive white noises environment in smart antenna, but also exactly estimate DOA values for near distance, low SNR and high correlated sources in mixed noises fields.

\section{CONFLICT OF INTEREST}

The authors confirm that this article content has no conflict of interest.

\section{ACKNOWLEDGEMENTS}

This work was supported by the MOE (Ministry of Education in China) Project of Humanities and Social Sciences (12YJCZH099), the Hunan Provincial Natural Science Foundation of China (Grant No. 2015JJ5025), the Natural Science Foundation of Jiangxi Province of China (20114BAB211017) and the science and technology plan project of Jiangxi Province (20122BBE500048).

\section{REFERENCES}

[1] R.M.Shubair, M.A.A.Qutayri, and J.M.Samhan, "A setup for the evaluation of MUSIC and LMS algorithms for a smart antenna system”, Journal of Communications, vol.2, no.4, pp.71-77, 2007.

[2] Md.Bakhar, and Dr.V.R.M.P.V.Hunagund, "Eigen str- ucture based direction of arrival estimation algorithms for smart antenna systems", International Journal of Computer Science and Network Security, vol.9, no.11, pp.96-100, 2009.

[3] S.Katariya, "A survey on smart antenna system", Internat- ional Journal of Electronics\&Communication Technology, vol.2, no.3, pp.123-126, 2011.

[4] S. C. Kim, I. Song, S. Yoon and S. R. Park, "DOA estim- ation of angle-perurbed sources for wireless mobile com- munications", $I E$ ICE Trans. Communication, vol. E83-B, no.11, pp.2537-2541, 2000.

[5] J.X.Wu, T.Wang, Z.Y.Suo, and Z. Bao, "DOA estimation for ULA by spectral Capon rooting method", Electronics Letters, vol.45, no.1, pp.84-85, 2009.

[6] J.M.Xin and A. Sane, "Linear prediction approach to direction estimation of cyclostationary signals in multipath environ- ment", IEEE Transactions on Signal Processing, vol.49, no.4, pp.710-720, 2001.

[7] E.Grosicki, K. Abed-Meraim, and K.Y.Hua, "A weighted linear prediction method for near-field source localization," IEEE Transactions on Signal Processing, vol.53, no.10, pp.3651-3660, 2005.

[8] T.B.Lavate, V.K.Kokate, and A.M.Sapkal, "Performance analysis of MUSIC and ESPRIT DOA estimation algorith- ms for adaptive array smart antenna in moblie communic- ation", International Journal of Computer Networks, vol.2, no.3, pp.152-158, 2010.

[9] D.F.Zha, and T.S.Qiu, "Direction finding in non-Gaussian impulsive noise environments", Digital Signal Processing, vol.17, no.2, pp.451-465, 2007. 
[10] K.Wang, Y.S.Zhang, and D. Shi, "Novel algorithm on DOA estimation for correlated sources under complex sy- mmetric toeplitz noise", Journal of Systems Engineering and Electronics, vol.19, no.5, pp.902-906, 2008.

[11] A.Kisliansky, R.Shavit, and J.Tabrikian, "Direction of arrival estimation in the presence of noise coupling in antenna arrays", IEEE Transactions On Antennas And Propagation, vol.55, no.7, pp.1940-1947, 2007.

[12] A.Thakre, M.Haardt, and K.Giridhar, "Singal snapshot spatial smoothing with improved effective array aperture", IEEE Signal Processing Letters, vol.16, no.6, pp505-508, 2009.

[13] Y.Zhang, Q.Wang, and A.M.Huang, "Localization of narr- ow band sources in the presence of mutual coupling via sparse solution finding", Progress in Electromagnetics Re- search, vol.86, pp.243257, 2008.
[14] C.R.Dongarsane, and A.N.Jadhav, "Simulation study on DOA estimation using MUSIC algorithm", International Journal of Technology and Engineering System, vol.2, no.1, pp.54-57, 2011.

[15] K.K.Kumari, B.Sudheer, and K.V.Suryakiran, "Algorithm for direction of arrival estimation in a smart antenna", International Journal of Communication Engineering Applications, vol.2, no.4, pp.144-149, 2011.

[16] A.Hirata, T.Morimoto, and Z.Kawasaki, "DOA estimation of ultawideband EM waves with MUSIC and interferom- etry", IEEE Antennas and Wireless Propagation Letters, vol.2, no.1, pp.190-193, 2003.

[17] F.Taga, "Smart Music algorithm for DOA estimation", Electronics Letters, vol.33, no.3, pp.190-191, 1997.

Received: May 26, 2015

Revised: July 14, 2015

Accepted: August 10,2015

(C) Bo et al.; Licensee Bentham Open.

This is an open access article licensed under the terms of the (https://creativecommons.org/licenses/by/4.0/legalcode), which permits unrestricted, noncommercial use, distribution and reproduction in any medium, provided the work is properly cited. 